\title{
Familial pulmonary arterial hypertension by KDR heterozygous loss of function
}

\author{
Mélanie Eyries ${ }^{1,2,13}$, David Montani $\mathbb{1}^{3,4,5,13}$, Barbara Girerd ${ }^{3,4}$, Nicolas Favrolt ${ }^{6}$, \\ Marianne Riou ${ }^{7}$, Laurence Faivre ${ }^{8}$, Grégoire Manaud ${ }^{5}$, Frédéric Perros ${ }^{5}$, \\ Stefan Gräf (10,10,11, Nicholas W. Morrell ${ }^{9,12}$, Marc Humbert (10) ${ }^{3,4,5}$ and \\ Florent Soubrier (10 ${ }^{1,2}$
}

Affiliations: ${ }^{1}$ Hôpital Pitié-Salpêtrière, Département de génétique, Assistance Publique-Hôpitaux de Paris, Paris, France. ${ }^{2}$ UMR S1166-ICAN, Sorbonne Université, INSERM, Paris, France. ${ }^{3}$ Univ. Paris-Sud, Faculté de Médecine, Université Paris-Saclay, Le Kremlin Bicêtre, France. ${ }^{4}$ Assistance Publique Hôpitaux de Paris, Service de Pneumologie, Centre de Référence de l'Hypertension Pulmonaire, Hôpital Bicêtre, Le Kremlin Bicêtre, France. ${ }^{5}$ UMR_S 999, Univ. Paris-Sud, INSERM, Hôpital Marie Lannelongue, Le Plessis Robinson, France. 'Service de Pneumologie et Soins Intensifs Respiratoires, Centre de référence constitutif des maladies pulmonaires rares de l'adulte, Centre de compétence de l'hypertension pulmonaire, CHU DijonBourgogne, Dijon, France. ${ }^{7}$ Service de pneumologie, Nouvel hôpital civil, Strasbourg. France. ${ }^{8}$ Centre de génétique, FHU TRANSLAD, Institut GIMI et UMR INSERM 1231, CHU de Dijon et Université de Bourgogne, Dijon, France. ${ }^{9}$ NIHR Bioresource - Rare Diseases, Cambridge Biomedical Campus, Cambridge, UK. ${ }^{10}$ Dept of Medicine, University of Cambridge, Cambridge Biomedical Campus, Cambridge, UK. ${ }^{11}$ Dept of Haematology, University of Cambridge, Cambridge Biomedical Campus, Cambridge, UK. ${ }^{12}$ Dept of Medicine, University of Cambridge School of Clinical Medicine, Addenbrooke's and Royal Papworth Hospitals, Cambridge, UK. ${ }^{13}$ Equally contributing authors.

Correspondence: Florent Soubrier, UMR_S 1166 ICAN, Paris 75634, France. E-mail: florent.soubrierQ sorbonne-universite.fr

@ERSpublications

$K D R$ mutations were identified in two families with a particular form of $\mathrm{PAH}$ characterised by low DLCOc and radiological evidence of parenchymal lung disease http://bit.ly/30npPPn

Cite this article as: Eyries M, Montani D, Girerd B, et al. Familial pulmonary arterial hypertension by KDR heterozygous loss of function. Eur Respir J 2020; 55: 1902165 [https://doi.org/10.1183/ 13993003.02165-2019].

ABSTRACT Beyond the major gene BMPR2, several new genes predisposing to PAH have been identified during the last decade. Recently, preliminary evidence of the involvement of the KDR gene was found in a large genetic association study.

We prospectively analysed the KDR gene by targeted panel sequencing in a series of $311 \mathrm{PAH}$ patients referred to a clinical molecular laboratory for genetic diagnosis of PAH.

Two index cases with severe PAH from two different families were found to carry a loss-of-function mutation in the KDR gene. These two index cases were clinically characterised by low diffusing capacity for carbon monoxide adjusted for haemoglobin $\left(D_{\mathrm{LCO}} \mathrm{c}\right)$ and interstitial lung disease. In one family, segregation analysis revealed that variant carriers are either presenting with $\mathrm{PAH}$ associated with low $D_{\mathrm{LCO}} \mathrm{c}$, or have only decreased $D_{\mathrm{LCO}} \mathrm{c}$, whereas non-carrier relatives have normal $D_{\mathrm{LCO}}$ c. In the second family, a single affected carrier was alive. His carrier mother was unaffected with normal $D_{\mathrm{LCO}}$.

We provided genetic evidence for considering $K D R$ as a newly identified PAH-causing gene by describing the segregation of $K D R$ mutations with PAH in two families. In our study, $K D R$ mutations are associated with a particular form of $\mathrm{PAH}$ characterised by low $D_{\mathrm{LCO}} \mathrm{C}$ and radiological evidence of parenchymal lung disease including interstitial lung disease and emphysema.

This article has supplementary material available from erj.ersjournals.com

This article has an editorial commentary: https://doi.org/10.1183/13993003.00326-2020

Received: 7 Nov 2019 | Accepted after revision: 2 Jan 2020

Copyright OERS 2020 


\section{Introduction}

Pulmonary arterial hypertension $(\mathrm{PAH})$ is a rare and devastating disease, resulting from progressive obliteration of small calibre pulmonary arteries by proliferating vascular cells and intimal fibrosis, and leading to cardiac failure. PAH can complicate well identified pathological conditions, or occur in the context of family history or genetic mutations causing heritable PAH (hPAH), or is considered as idiopathic (iPAH) in the absence of an identified predisposing factor [1]. The major gene associated with PAH is $B M P R 2$, encoding a type 2 receptor of the bone morphogenic protein (BMP) signalling pathway. During the last decade, several new genes have been identified making PAH a heterogeneous genetic disease [2]. The initial discovery of $B M P R 2$, exploited the collection of informative multiplex PAH families and the implication of other genes belonging to the BMP signalling pathway was based on a candidate gene approach, such as $A C V R L 1$ and $E N G$ in rare cases of hereditary haemorrhagic telangiectasia-associated PAH, SMAD9 or BMP10 [2, 3]. The TBX4 gene was identified owing to large genomic deletions encompassing the gene and was later shown to be a major predisposing gene for hPAH [4-6].The recently identified genes were the results of whole exome or genome sequencing in families or large cohorts of sporadic cases and included ATP13A3, GDF2 (BMP9), SOX17, AQP1, KCNK3 and CAV1 [6-9]. EIF2AK4, the gene responsible for heritable pulmonary veno-occlusive disease (hPVOD) was also identified by exome sequencing [10]. Following initial gene identification, further investigation of these genes in larger populations of patients gave contrasted results because no other case was identified for some of them, while for others, such as GDF2, several pathogenic variants were found in PAH cases [11].

In the course of a genetic association study using whole genome sequencing in a large series of PAH cases and controls, GRÄF et al. [6] found preliminary evidence for a possible involvement of kinase insert domain receptor $(K D R)$ gene in $\mathrm{PAH}$ by comparing the number of protein-truncating variants within the gene in patients and controls. However, this finding did not achieve genome-wide significance in that analysis and therefore was not considered definitive.

The KDR gene encodes the receptor for vascular endothelial growth factor (VEGF)-A, VEGFR2, that transduces the signal for most of VEGF effects on endothelial cells during development and in adult. Indeed, activation of VEGFR2 through ligand binding stimulates several signalling pathways including the extracellular signal-regulated kinases (ERK)1/2 pathway which enhances cell proliferation, the PI3 kinaseprotein kinase $\mathrm{B}(\mathrm{PKB})$ pathway which promotes cell survival, and the p38 mitogen-activated protein kinase (MAPK) and C-terminal Src kinase (CSK; Src) which induces cell migration [12]. In addition, CSK (Src) activation and PI3K-PKB can induce nitric oxide (NO) production and, hence, cell permeability that may promote angiogenesis [13]. VEGF is highly expressed in the lung and VEGF signalling plays an essential role during fetal lung development and serves as a maintenance factor during adult life [14]. Moreover, an antagonist of VEGFR2, SU5416 (Sugen), led to lung immaturity in rats [15] and was shown to induce pulmonary hypertension in association with hypoxia in adult rats [16].

In view of the prominent role of VEGFR2 in lung vasculature and preliminary evidence obtained from the human PAH genetic study [6], we prospectively analysed the KDR gene by targeted panel sequencing in a series of $311 \mathrm{PAH}$ patients referred to a clinical molecular laboratory for genetic diagnosis of PAH. We report the genotype and phenotype characteristics of two families carrying a KDR loss of function variant.

\section{Patients and methods}

Genetic analysis

Clinical molecular diagnosis by next-generation sequencing (NGS)-based gene panel on hPAH and hPVOD predisposing genes and NGS data analysis was performed as previously described in EYRIES et al. [3]. Briefly, for all incident index cases referred to the laboratory, a custom gene panel including nine established PAH and PVOD genes (BMPR2, TBX4, EIF2AK4, CAV1, KCNK3, SMAD9, ACVRL1, ENG, $B M P 9)$ as well as research genes, including the KDR gene, was used. Splicing variants were analysed by splicing prediction tools, MaxEntScan, NNSplice, GeneSplicer and HumanSplicingFinder through Alamut integrator software (https://blog.goldenhelix.com/splice-site-algorithms/). Thus $311 \mathrm{PAH}$ patients followed within the French Pulmonary Hypertension Network were explored between 2017 and 2019 for KDR mutations. All patients underwent genetic counselling and signed written informed consent for genetic analysis. KDR DNA sequence was compared with the reference sequence (NM_002253.2). Likely gene disrupting (LGD) variants of KDR were confirmed by Sanger sequencing. Variant classification follows criteria adopted by the American College of Medical Genetics (ACMG) [17]

\section{Clinical assessment}

Routine evaluation at diagnosis of PAH included medical history, physical examination, assessment of dyspnoea by New York Heart Association (NYHA) functional class, 6-min walk distance (6MWD), echocardiography, pulmonary function tests, contrast-enhanced computed tomography (CT) of the chest, 
ventilation/perfusion lung scan, abdominal ultrasound, autoimmunity screening and HIV serology. Pulmonary function tests included forced expiratory volume in $1 \mathrm{~s}\left(\mathrm{FEV}_{1}\right)$, forced vital capacity (FVC), $\mathrm{FEV}_{1} / \mathrm{FVC}$ ratio, total lung capacity (TLC) and diffusing capacity for carbon monoxide adjusted for hemoglobin $\left(D_{\mathrm{LCO}}\right)$. Haemodynamic measurements were obtained by right heart catheterisation according to routine protocols including right atrial pressure (RAP), mean pulmonary artery pressure (mPAP), pulmonary artery wedge pressure (PAWP). Cardiac output was measured by the thermodilution technique and cardiac index was calculated as cardiac output/body surface area. Pulmonary vascular resistance (PVR) was calculated as (mPAP-PAWP)/cardiac output (expressed in Wood units (WU)).

Clinical characteristics at diagnosis and follow-up were stored in the Registry of the French Pulmonary Hypertension Network (French Referral Center, Hospital Kremlin-Bicêtre and 25 associated centres spread across France). This Registry was set up in agreement with French bioethics laws (Commission Nationale de l'Informatique et des Libertés).

\section{Results}

Two index PAH patients from two different families were found to carry a KDR loss of function variant. Their families were explored subsequently (figure 1).

\section{Family 1}

Index case (II.4) was a 55 year-old woman diagnosed with PAH. She had no medical history and no tobacco exposure. Her family history includes an unexplained death of her sister at 46 years (II.2) due to cardio-respiratory insufficiency in a context of dexfenfluramine intake (figure 1a). At diagnosis, she was in NYHA functional class III and 6MWD was $280 \mathrm{~m}$. Pulmonary function tests showed mild restriction $\left(\mathrm{FEV}_{1} 70 \%, \mathrm{FVC} 74 \%, \mathrm{FEV}_{1} / \mathrm{FVC} 0.8\right.$, TLC $\left.79 \%\right)$ and a significant decrease in $D_{\mathrm{LCO}}$ at $29 \%$ of predicted values. Arterial blood gas on room air revealed severe hypoxaemia with an arterial oxygen tension $\left(P_{\mathrm{aO}_{2}}\right)$ of $55 \mathrm{mmHg}$. N-terminal pro-brain natriuretic peptide (NT-proBNP) was at $664 \mathrm{pg} \cdot \mathrm{mL}^{-1}$. Echocardiography and cardiac magnetic resonance imaging (MRI) showed severe pulmonary hypertension $(\mathrm{PH})$ with right ventricular (RV) dilatation (diastolic RV volume $145 \mathrm{~mL}$ ) and dysfunction (RV ejection fraction 28\%) associated with paradoxical septum. There was no argument for chronic left heart disease or congenital heart disease. Right heart catheterisation at rest revealed severe pre-capillary pulmonary hypertension with a mPAP at $71 \mathrm{mmHg}$, a PAWP of $9 \mathrm{mmHg}$, cardiac index of $2.23 \mathrm{~L} \cdot \mathrm{min}^{-1} \cdot \mathrm{m}^{-2}$ and PVR of 15.7 WU. Acute vasodilatory test was negative. CT of the chest demonstrated mild interstitial lung disease with an upper-lobe predominance (figure $2 \mathrm{a}$ and $\mathrm{b}$ ). Dual oral combination therapy (endothelin receptor antagonist (ERA) and type 5 phosphodiesterase inhibitor (PDE5i) was initiated. At 18 months, clinical and haemodynamic status improved (NYHA functional class II, 6MWD $355 \mathrm{~m}$, mPAP $43 \mathrm{mmHg}$, cardiac index $3.3 \mathrm{~L} \cdot \mathrm{min}^{-1} \cdot \mathrm{m}^{-2}$ and PVR $\left.6.7 \mathrm{WU}\right)$.

Genetic analysis identified a heterozygous $K D R$ truncating mutation (c.3302T>G, p.Leu1101*) located in exon 24 of the gene, that induces a loss of the catalytic kinase domain of the receptor [18]. No other pathological mutation was identified on established PAH genes. Five other available family members were then genotyped. This KDR mutation was identified in one brother (II.1) who complained about dyspnoea for several years. Following KDR mutation identification, complete assessment revealed a diagnosis of PAH with moderate haemodynamic impairment (mPAP $27 \mathrm{mmHg}$, PAWP $4 \mathrm{mmHg}$, cardiac index $2.3 \mathrm{~L} \cdot \mathrm{min}^{-1} \cdot \mathrm{m}^{-2}$ and PVR 5.4 WU). He was in class III, and the 6-MWD was $400 \mathrm{~m}$ and NT-proBNP was normal. Pulmonary function tests were normal except for a significant decrease in $D_{\mathrm{LCO}} \mathrm{c}$ at $35 \%$. Arterial blood gas on room air revealed severe hypoxaemia with a $P_{\mathrm{aO}_{2}}$ of $69 \mathrm{mmHg}$. CT of the chest demonstrated interstitial lung disease including subpleural reticulation, septal lines and bronchiolar abnormalities (figure $2 \mathrm{c}$ and $\mathrm{d}$ ). Bronchoalveolar lavage was normal. A treatment by ERA and PDE5i combination therapy was initiated and the patient was alive after 6 months of evaluation.

Genetic analysis also identified three asymptomatic mutation carriers (III.2, III.4 and III.5 with ages of 46, 33 and 28 years, respectively). Clinical evaluation was proposed to asymptomatic carrier relatives. Pulmonary function tests identified a low $D_{\mathrm{LCOC}}$ in all carriers of the KDR mutation with $53 \%, 64 \%$ and $50 \%$ in individuals III.2, III.4 and III.5, respectively. $D_{\text {LCOC }}$ was normal in relative III.1 not carrying the familial mutation. CT of the chest was normal in III.2, and III.4 and identified a double aortic arch in III.5 (figure 2e). Relative III.5 performed a right heart catheterisation revealing no pulmonary hypertension (mPAP $17 \mathrm{mmHg}$ ) but a mild increase in PVR of unknown significance at $2.1 \mathrm{WU}$.

\section{Family 2}

The index case (III.3) is a 31 year old male diagnosed with PAH at the age of 30 years. Personal history reveals non-allergic asthma in childhood and minor former tobacco exposure (2 pack-years). His family history includes the death at 60 years of his maternal grandfather in a context of dyspnoea and right heart 


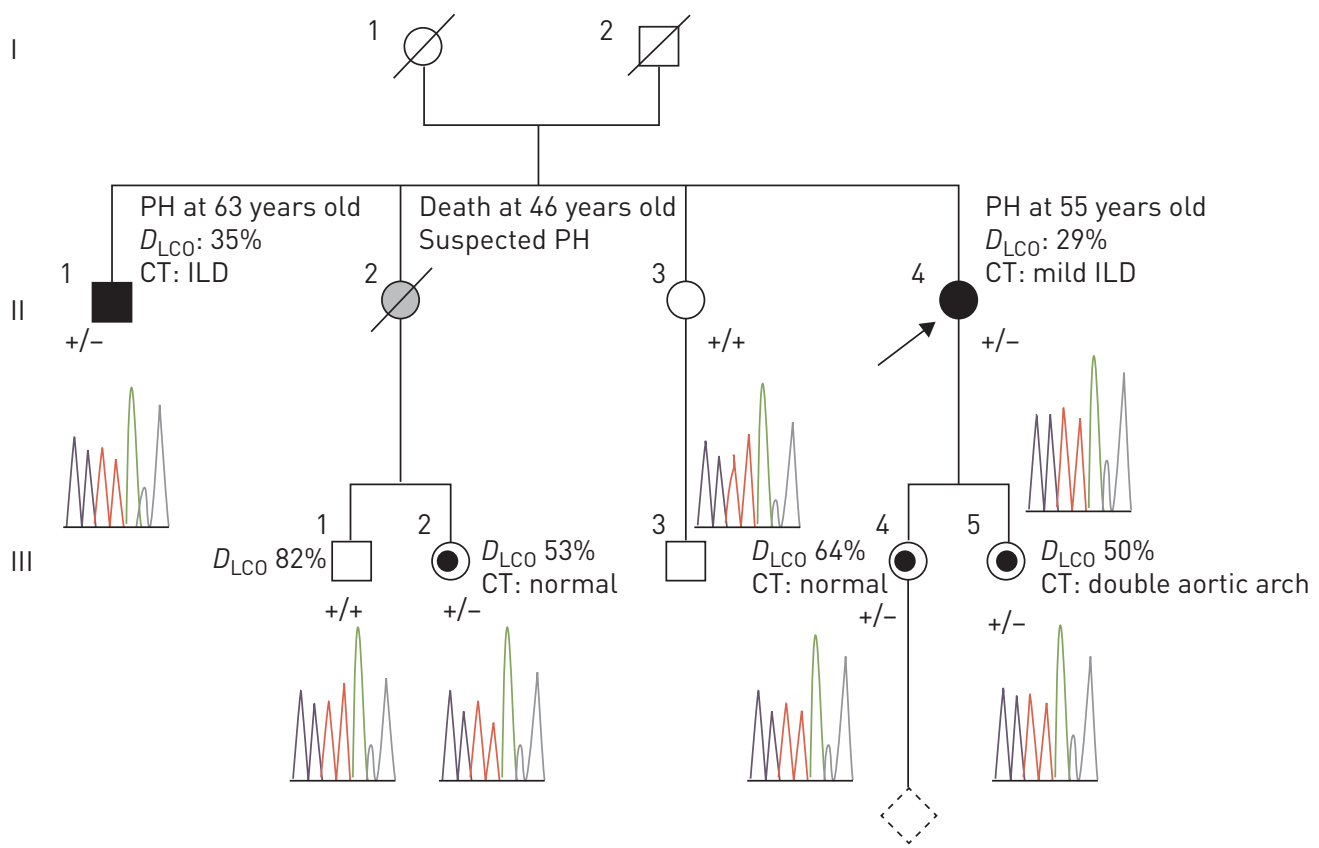

b)

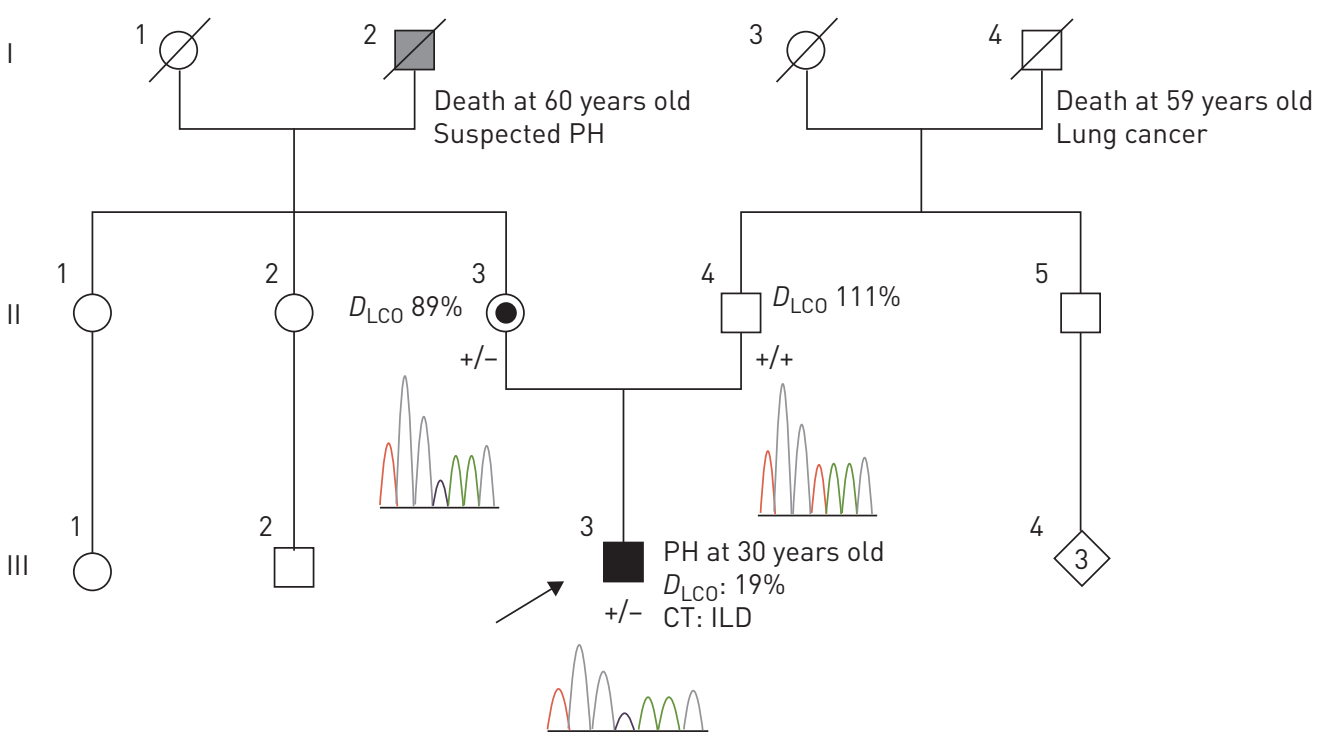

FIGURE 1 Family tree of carriers of KDR mutation: a) family 1 ; b) family 2 . +/+: mutation non-carrier. +/-: heterozygous carrier of a KDR mutation. $\square$ : indicate affected subjects. $\varnothing \square$ : indicate subjects with suspected PH. - indicates asymptomatic carriers of the familial KDR mutation.

failure (figure $1 \mathrm{~b}$ ). At diagnosis, he was in NYHA functional class III and 6MWD was $210 \mathrm{~m}$. Pulmonary function tests were normal contrasting with a major decrease in $D_{\mathrm{LCO}} \mathrm{C}$ at $19 \%$. Arterial blood gas on room air revealed severe hypoxaemia with a $P_{\mathrm{aO}}$ at $61 \mathrm{mmHg}$. Echocardiography and cardiac MRI showed RV dilatation (diastolic RV volume $218 \mathrm{~mL}$ ) and dysfunction (RV ejection fraction 31\%) associated with paradoxical septum. There was no argument for chronic left heart disease or congenital heart disease. Right heart catheterisation showed severe precapillary pulmonary hypertension (mPAP $48 \mathrm{mmHg}$, PAWP $3 \mathrm{mmHg}$, cardiac index $2 \mathrm{~L} \cdot \mathrm{min}^{-1} \cdot \mathrm{m}^{-2}$ and PVR $13.6 \mathrm{WU}$ ). Acute vasodilator testing was negative. CT of the chest identified interstitial lung disease including reticulations, septal lines and ground glass opacities, mild emphysema and mediastinal enlarged lymph nodes (figure $2 \mathrm{f}$ and $\mathrm{g}$ ). A positron emission tomography (PET)-CT identified mediastinal lymph node enlargement with moderate fixation (maximum standardised uptake value of 6.8) (figure 2h). Bronchoalveolar lavage was performed showing haemosiderin-laden macrophages (Golde score at 135). A diagnosis of sarcoidosis was initially suspected, 

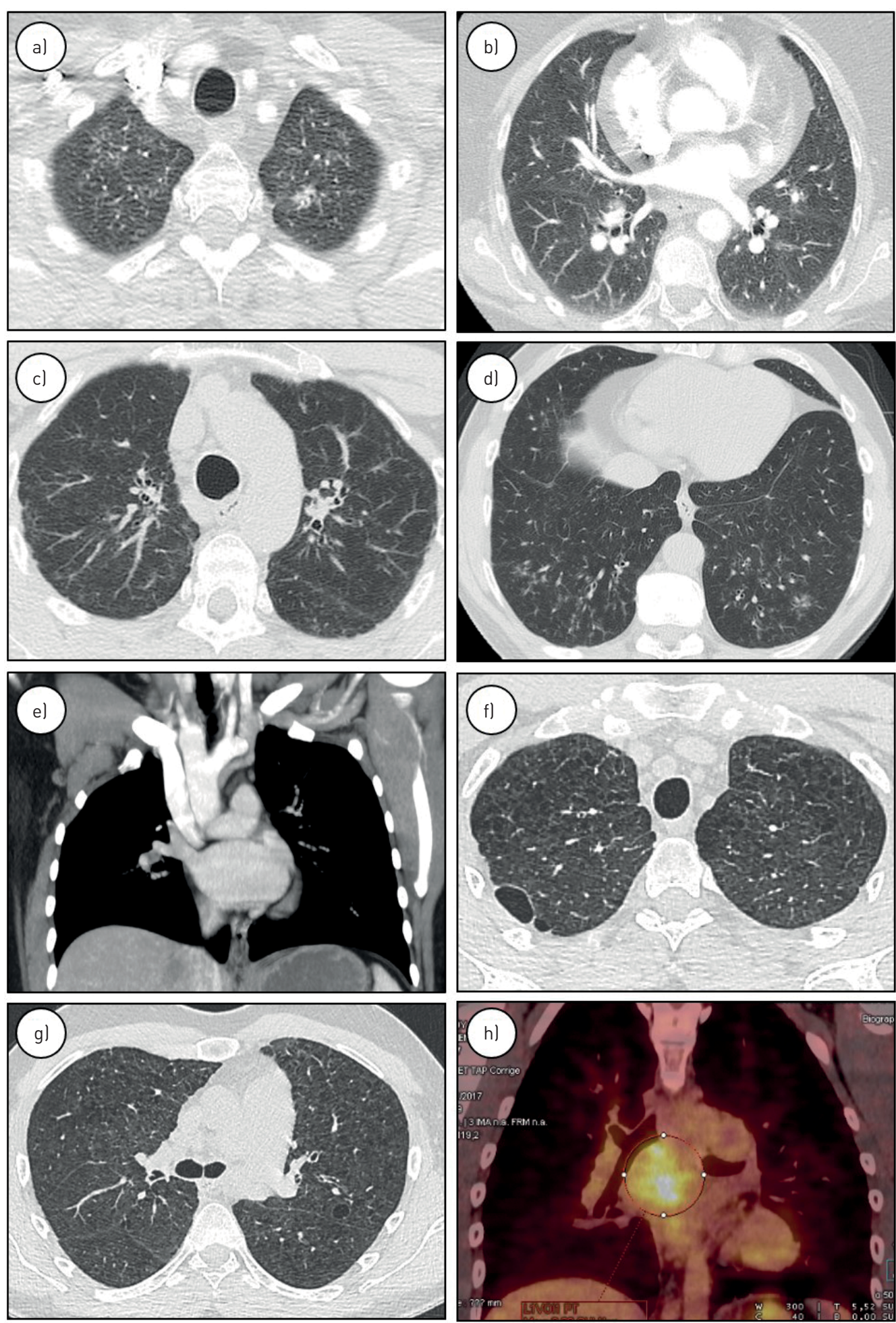

FIGURE 2 Computed tomography (CT) of the chest of KDR mutation carriers. a and b) CT of the chest in pulmonary arterial hypertension (PAH) patient II.4 from family 1 revealing interstitial lung disease with reticulations in upper lobes (a) and ground-glass opacities predominantly in the mid-lower lung (b). c and d) CT of the chest in PAH patient II.1 from family 1 revealing interstitial lung disease with subpleural reticulations (c) and bronchiolar lesions in the lower lungs (d). e) CT of the chest identified a double aortic arch in asymptomatic relative III.5 carrying a KDR mutation from family 1. f-h) In PAH patient III.3 from family 2, CT of the chest revealed reticulations, mild emphysema ( $f$ and $g$ ) and mediastinal lymph node enlargement with moderate fixation on positron emission tomography $\mathrm{CT}(\mathrm{h})$. 
and the patient received corticosteroid during 3 months without any improvement. Dual oral combination therapy (ERA and PDE5i) was then initiated. While there was a clinical stability (NYHA functional class II, $6 \mathrm{MWD}$ of $450 \mathrm{~m}$ ) for 2 years, the last right heart catheterisation showed a significant decrease of cardiac output and an increase of PVR to 11.1 WU. Listing for lung transplantation is currently discussed for this patient.

Genetic analysis was negative for the established genes involved in the development of PAH, but identified a heterozygous $K D R$ mutation (c.976+2T>C) located in the consensus splicing donor site in intron 7 of the gene. In silico analysis of this splicing variant showed that it is predicted to abolish the donor-splicing site of intron 7 leading to the skipping of exon 7 and a truncated protein p.His267Lysfs ${ }^{\star} 37$. Genotyping of both parents revealed that this mutation was inherited from the mother who had normal pulmonary function tests, normal CT of the chest and a normal $D_{\mathrm{LCO}}$ at $89 \%$. The maternal grandfather had died at the age of 60 with a clinical presentation consistent with unrecognised $\mathrm{PH}$.

\section{Discussion}

We described a new form of hPAH linked to KDR loss-of-function pathogenic variants on a single allele of the gene. We identified two index cases carrying KDR mutations after prospectively screening a series of 311 unrelated patients referred for PAH genetic investigation ( $0.6 \%$ of patients). In the first family, we identified a KDR truncating variant encoding a VEGFR2 molecule predicted to be devoid of tyrosine kinase catalytic site and of mapped key catalytic residues, such as tyrosine 1175, for autophosphorylation [18, 19]. Segregation analysis was possible on two generations and revealed that variant carriers are either presenting with declared PAH associated with low $D_{\mathrm{LCO}} \mathrm{c}$, or have only a decreased $D_{\mathrm{LCO}}$ without other symptoms, whereas non-carriers relatives are symptom free and have normal $D_{\mathrm{LCO}} \mathrm{c}$. In the second family, a splice variant that affects the consensus donor site was identified in KDR and is predicted to induce a truncation of the protein by skipping of exon 7 starting from the immunoglobulin-like domains 3 of VEGFR2 that was shown to be essential for VEGF binding [20], until the carboxy-terminal end of the protein. $D_{\mathrm{LCO}}$ was also deeply decreased in the affected index case whereas it was normal in the unaffected carrier mother of the PAH patient. Interestingly, in this family, the medical history of the maternal grandfather is consistent with $\mathrm{PAH}$-induced right heart failure and death. Thus, we identified two distinct variants classified as pathogenic according to ACMG criteria in the KDR gene [17]. KDR loss of function variants are extremely rare in the general population ( 11 observed variants in the 141456 individuals available in gnomAD database) and this gene has a pLI score of $1(\mathrm{o} / \mathrm{e}=0.15(0.09-0.25), \% \mathrm{HI}$ : 2.22) meaning that it is extremely intolerant to loss of function $[21,22]$. These findings are strongly supported by a parallel study of the International Consortium for Genetic Studies in PAH analysing whole-genome sequencing data of 13,037 individuals including 1,148 subjects recruited to the PAH domain [23].

The $D_{\mathrm{LCO}} \mathrm{c}$ decrease is more pronounced in affected subjects than in non-affected carriers, and whether $D_{\mathrm{LCO}}$ decrease is an early marker of the development of $K D R$-associated disease needs to be further investigated. The long-term follow-up of the reported carrier subjects will be informative if the pathological process cannot be halted. In addition to low $D_{\mathrm{LCO}} \mathrm{c}$, all affected patients presented with radiological evidence of interstitial lung disease on high-resolution CT that can be prominent and led to an initial erroneous diagnosis of sarcoidosis in one patient. Conversely, KDR mutation carriers without declared PAH have normal high-resolution CT even if subclinical lung parenchymal involvement might be suspected in view of $D_{\mathrm{LCO}} \mathrm{C}$ decrease. Therefore, presence of mild interstitial lung disease could be an important radiological marker of $\mathrm{PAH}$ development in KDR mutation carriers. In this context, the $D_{\mathrm{LCO}}$ decrease reflects gas exchange impairment that may be the consequence of lung parenchymal involvement and of pulmonary distal vascular bed remodelling. Ventilation/perfusion lung scan of the three PAH patients and CT pulmonary angiogram (performed in patients II.4 family 1 and III.3 family 2) found no evidence of significant pulmonary vasculature malformations. However, these examinations explore segmental and subsegmental pulmonary arteries, and cannot exclude developmental abnormalities of pulmonary microvasculature. The association of parenchymal lung disease and pulmonary hypertension may suggest a possible developmental lung disorders rather than a specific and unique pulmonary vascular remodelling as observed in $\mathrm{hPAH}$ due to $B M P R 2$ mutation. However, the absence of available lung tissue from affected subjects with KDR mutations, is limiting the pathophysiological interpretation that could be drawn from observed lesions.

The expression of $K D R$ is high in human embryonic lung endothelial cells [24] and the absolute requirement of VEGFR2 for development of the embryonic vasculature in mice has been demonstrated by KDR inactivation which produces an embryonic lethal phenotype with abnormal blood vessel development [25]. During rat development, VEGF mRNA is expressed in distal airway epithelial cells and the sub-epithelial matrix of E11.5 lungs, whereas VEGFR2-positive cells were localised in the mesenchyme closely apposed to the developing epithelium $[26,27]$. This spatial relationship between epithelial cells which produce VEGF 
and endothelial cells that express VEGFR2 enables an essential cross-talk for the formation of the epithelium-capillary bilayers, which are essential for gas exchange. VEGF signalling has a major role for proper blood vessel formation in the embryonic lung but also after birth. JAKKULA et al. [15] used the VEGF-R2 antagonist SU5416 (Sugen) to show that, in the neonatal rat, inhibition of VEGF signalling reduces alveolarisation and decreases arterial density, showing that VEGFR2 contributes significantly to vascular growth after birth where a rapid lung growth occurs. Indeed, premature infants with lung hypoplasia have distal air spaces that fail to septate, leading to a reduced surface area for gas exchange [28]. Chronic treatment of adult rats with SU5416 induced alveolar septal cell apoptosis and leads to the enlargement of air spaces indicative of emphysema, suggesting that VEGF signalling is required for the maintenance of the pulmonary vasculature and alveolar structure during adulthood $[29,30]$.

Loss of function of a single allele of KDR is expected to decrease VEGFR2 mediated signalling of VEGF through a mechanism of haplo-insufficiency that has already been shown for PAH due to mono-allelic loss of function of BMPR2 [31]. Indeed, in mice carrying a heterozygous disruption of $K D R$, the $K D R$ mRNA was almost 50\% reduced and an abnormal response to ischaemic preconditioning of the heart was observed [32]. Moreover, heterozygous mice for KDR loss of function display profound defects in supporting tumor growth and angiogenesis, clearly demonstrating a gene dosage effect of this gene and supporting a pathogenic role in humans [33].

Although a partially deficient KDR gene as a cause of PAH may appear paradoxical in view of the increased plasma VEGF concentration and the VEGF and KDR expression in PAH lesions ([34], and our results in supplementary figure S1), further elements supporting the pathogenic role of decrease VEGF signalling by KDR loss of function in human PAH comes from the experimental model of rat PAH caused by SU5416. PAH occurs in rats after SU5416 administration in combination with hypoxia, or unilateral pneumonectomy or in immuno-deficient animals and lung lesions are close to those observed in human PAH $[16,35,36]$. These animal models clearly support a maintenance role of VEGF signalling in adulthood and VEGF signalling antagonises endothelial apoptosis, a phenomenon that is proposed to be involved in the SU5416 model. These models are, however, different from the KDR haplo-insufficiency induced form of human $\mathrm{PAH}$, since the deficiency is only partial (around 50\% of expression) and is acting from developmental life where lung vascular restriction by diminished VEGF signalling might be important.

Since decreased $D_{\mathrm{LCOC}}$ seems to be the only detectable physiological abnormality in unaffected KDR mutation carriers, another triggering factor needs to be hypothesised to explain the disease initiation. CT of the chest from affected subjects, together with worsened $D_{\mathrm{LCO}} \mathrm{c}$, are suggestive of parenchymal lung disease. Experimental models have documented the effects of VEGF signalling in the bleomycin model of fibrosis. Indeed, VEGF signalling has other functions beyond angiogenesis, such as stimulation of epithelial proliferation, prevention of epithelial apoptosis and attenuation of vascular remodelling via protective effects on endothelial cells in a context of experimental lung fibrosis in rats $[29,37,38]$.

As for all newly discovered gene involvement in hereditary disease, the central question is whether the disease can be imputed to these mutations. We identified loss of function variants in the KDR gene, for which loss of function variants are extremely rare in the general population. We have also clearly shown in one family the cosegregation of the pathogenic variant with a specific phenotype combining PAH and ILD associated with a decrease in $D_{\mathrm{LCO}} \mathrm{c}$. VEGFR2 is known to be involved in the development of the lung vasculature and plays a central role in the structural specificity of the lung enabling gas exchange. In addition, VEGF signalling plays a major role in maintaining epithelial and endothelial integrity. Thus, previous pathophysiological and WGS data, together with clinical and genetic elements from this study allow to consider KDR as a new PAH causing gene. Whether KDR linked PH should be considered as a pure heritable $\mathrm{PAH}$ form or as $\mathrm{PH}$ occurring in the setting of developmental lung disorder should be discussed in the light of lung tissue analysis from affected subjects and from the murine model of KDR haploinsufficiency [39].

Acknowledgements: This study makes use of data generated by the DECIPHER community. A full list of centres who contributed to the generation of the data is available from http://decipher.sanger.ac.uk and via email from decipher@sanger.ac.uk. We thank Anne Leroy and Marie-Christine Waill for expert technical assistance.

Conflict of interest: M. Eyries has nothing to disclose. D. Montani reports grants and personal fees from Actelion and Bayer, personal fees from GSK, Pfizer and MSD, outside the submitted work. B. Girerd has nothing to disclose. N. Favrolt has nothing to disclose. M. Riou has nothing to disclose. L. Faivre has nothing to disclose. G. Manaud has nothing to disclose. F. Perros has nothing to disclose. S. Gräf has nothing to disclose. M.W. Morrell is an employee of Morphogen-IX. M. Humbert reports personal fees from Acceleron, Actelion, Merck and United Therapeutics, grants and personal fees from Bayer and GSK, outside the submitted work. F. Soubrier has nothing to disclose.

Support statement: Funding for the project was provided by the Wellcome Trust. Funding information for this article has been deposited with the Crossref Funder Registry. 


\section{References}

1 Humbert M, Guignabert C, Bonnet S, et al. Pathology and pathobiology of pulmonary hypertension: state of the art and research perspectives. Eur Respir J 2019; 53: 1801887.

2 Machado RD, Southgate L, Eichstaedt CA, et al. Pulmonary arterial hypertension: a current perspective on established and emerging molecular genetic defects. Hum Mutat 2015; 36: 1113-1127.

3 Eyries M, Montani D, Nadaud S, et al. Widening the landscape of heritable pulmonary hypertension mutations in paediatric and adult cases. Eur Respir J 2019; 53: 1801371.

4 Kerstjens-Frederikse WS, Bongers EMHF, Roofthooft MTR, et al. TBX4 mutations (small patella syndrome) are associated with childhood-onset pulmonary arterial hypertension. J Med Genet 2013; 50: 500-506.

5 Levy M, Eyries M, Szezepanski I, et al. Genetic analyses in a cohort of children with pulmonary hypertension. Eur Respir J 2016; 48: 1118-1126.

6 Gräf S, Haimel M, Bleda M, et al. Identification of rare sequence variation underlying heritable pulmonary arterial hypertension. Nat Commun 2018; 9: 1416.

$7 \quad$ Ma L, Roman-Campos D, Austin ED, et al. A novel channelopathy in pulmonary arterial hypertension. $N$ Engl $J$ Med 2013; 369: 351-361.

8 Austin ED, Ma L, LeDuc C, et al. Whole exome sequencing to identify a novel gene (caveolin-1) associated with human pulmonary arterial hypertension. Circ Cardiovasc Genet 2012; 5: 336-343.

9 Wang G, Fan R, Ji R, et al. Novel homozygous BMP9 nonsense mutation causes pulmonary arterial hypertension: a case report. BMC Pulm Med 2016; 16: 17.

10 Morrell NW, Aldred MA, Chung WK, et al. Genetics and genomics of pulmonary arterial hypertension. Eur Respir J 2019; 53: 1801899.

11 Wang X-J, Lian T-Y, Jiang X, et al. Germline BMP9 mutation causes idiopathic pulmonary arterial hypertension. Eur Respir J 2019; 53: 1801609.

12 Ferrara N. Vascular endothelial growth factor: basic science and clinical progress. Endocr Rev 2004; 25: 581-611.

13 Liao W, Feng L, Zheng J, et al. Deciphering mechanisms controlling placental artery endothelial cell migration stimulated by vascular endothelial growth factor. Endocrinology 2010; 151: 3432-3444

14 Voelkel NF, Vandivier RW, Tuder RM. Vascular endothelial growth factor in the lung. Am J Physiol Lung Cell Mol Physiol 2006; 290: L209-L221.

15 Jakkula M, Le Cras TD, Gebb S, et al. Inhibition of angiogenesis decreases alveolarization in the developing rat lung. Am J Physiol Lung Cell Mol Physiol 2000; 279: L600-L607.

16 Taraseviciene-Stewart L, Kasahara Y, Alger L, et al. Inhibition of the VEGF receptor 2 combined with chronic hypoxia causes cell death-dependent pulmonary endothelial cell proliferation and severe pulmonary hypertension. Faseb J 2001; 15: 427-438.

17 Richards S, Aziz N, Bale S, et al. ACMG Laboratory Quality Assurance Committee. Standards and guidelines for the interpretation of sequence variants: a joint consensus recommendation of the American College of Medical Genetics and Genomics and the Association for Molecular Pathology. Genet Med 2015; 17: 405-424.

18 Terman BI, Dougher-Vermazen M, Carrion ME, et al. Identification of the KDR tyrosine kinase as a receptor for vascular endothelial cell growth factor. Biochem Biophys Res Commun 1992; 187: 1579-1586.

19 Takahashi T, Yamaguchi S, Chida K, et al. A single autophosphorylation site on KDR/Flk-1 is essential for VEGF-A-dependent activation of PLC-gamma and DNA synthesis in vascular endothelial cells. EMBO J 2001; 20: 2768-2778.

20 Shinkai A, Ito M, Anazawa $\mathrm{H}$, et al. Mapping of the sites involved in ligand association and dissociation at the extracellular domain of the kinase insert domain-containing receptor for vascular endothelial growth factor. $J$ Biol Chem 1998; 273: 31283-31288.

21 Firth HV, Richards SM, Bevan AP, et al. DECIPHER: Database of Chromosomal Imbalance and Phenotype in Humans Using Ensembl Resources. Am J Hum Genet 2009; 84: 524-533.

22 Lek M, Karczewski KJ, Minikel EV, et al. Analysis of protein-coding genetic variation in 60,706 humans. Nature 2016; 536: 285-291.

23 Swietlik EM, Greene D, Zhu N, et al. Reduced transfer coefficient of carbon monoxide in pulmonary arterial hypertension implicates rare protein-truncating variants in KDR. bioRxiv 2019; preprint [https://doi.org/10.1101/ 2019.12.11.871210].

24 Kaipainen A, Korhonen J, Pajusola K, et al. The related FLT4, FLT1, and KDR receptor tyrosine kinases show distinct expression patterns in human fetal endothelial cells. J Exp Med 1993; 178: 2077-2088.

25 Shalaby F, Ho J, Stanford WL, et al. A requirement for Flk1 in primitive and definitive hematopoiesis and vasculogenesis. Cell 1997; 89: 981-990.

26 Healy AM, Morgenthau L, Zhu X, et al. VEGF is deposited in the subepithelial matrix at the leading edge of branching airways and stimulates neovascularization in the murine embryonic lung. Dev Dyn 2000; 219: 341-352.

27 Gebb SA, Shannon JM. Tissue interactions mediate early events in pulmonary vasculogenesis. Dev Dyn 2000; 217 : 159-169.

28 Mitchell SH, Teague WG. Reduced gas transfer at rest and during exercise in school-age survivors of bronchopulmonary dysplasia. Am J Respir Crit Care Med 1998; 157: 1406-1412.

29 Kasahara Y, Tuder RM, Taraseviciene-Stewart L, et al. Inhibition of VEGF receptors causes lung cell apoptosis and emphysema. J Clin Invest 2000; 106: 1311-1319.

30 Kojonazarov B, Hadzic S, Ghofrani HA, et al. Severe emphysema in the SU5416/hypoxia rat model of pulmonary hypertension. Am J Respir Crit Care Med 2019; 200: 515-518.

31 Machado RD, Pauciulo MW, Thomson JR, et al. BMPR2 haploinsufficiency as the inherited molecular mechanism for primary pulmonary hypertension. Am J Hum Genet 2001; 68: 92-102.

32 Thirunavukkarasu M, Addya S, Juhasz B, et al. Heterozygous disruption of Flk-1 receptor leads to myocardial ischaemia reperfusion injury in mice: application of affymetrix gene chip analysis. J Cell Mol Med 2008; 12: 1284-1302.

33 Oladipupo SS, Kabir AU, Smith C, et al. Impaired tumor growth and angiogenesis in mice heterozygous for Vegfr2 (Flk1). Sci Rep 2018; 8: 14724.

34 Tuder RM, Chacon M, Alger L, et al. Expression of angiogenesis-related molecules in plexiform lesions in severe pulmonary hypertension: evidence for a process of disordered angiogenesis. J Pathol 2001; 195: 367-374. 
35 Toba M, Alzoubi A, O’Neill KD, et al. Temporal hemodynamic and histological progression in Sugen5416/ hypoxia/normoxia-exposed pulmonary arterial hypertensive rats. Am J Physiol Heart Circ Physiol 2014; 306: $\mathrm{H} 243-\mathrm{H} 250$.

36 Happé CM, de Raaf MA, Rol N, et al. Pneumonectomy combined with SU5416 induces severe pulmonary hypertension in rats. Am J Physiol Lung Cell Mol Physiol 2016; 310: L1088-L1097.

37 Roberts JR, Perkins GD, Fujisawa T, et al. Vascular endothelial growth factor promotes physical wound repair and is anti-apoptotic in primary distal lung epithelial and A549 cells. Crit Care Med 2007; 35: 2164-2170.

38 Farkas L, Farkas D, Ask K, et al. VEGF ameliorates pulmonary hypertension through inhibition of endothelial apoptosis in experimental lung fibrosis in rats. J Clin Invest 2009; 119: 1298-1311.

39 Simonneau G, Montani D, Celermajer DS, et al. Haemodynamic definitions and updated clinical classification of pulmonary hypertension. Eur Respir J 2019; 53: 1801913. 